\title{
Experimental Determination of the Form and Structure Factor of Molten Lithium
}

\author{
H. Olbrich, H. Ruppersberg *, and S. Steeb \\ Max-Planck-Institut für Metallforschung, Institut für Werkstoffwissenschaften, Stuttgart
}

Z. Naturforsch. 38 a, 1328-1336 (1983); received August 3, 1983

The aim of the present paper is to state differences in the spatial distribution of valency electrons for free metal atoms and for molten metals. The element lithium was chosen since both its inner and outer electrons play an important role, so that when a precise X-ray and a precise neutron-diffraction experiment have been carried out a difference, if it exists at all, should be recognizable. The correction of the X-ray data for inelastic, i.e. Compton scattering was done on the basis of an inelastic measurement performed on a spectrometer equipped with a position sensitive detector.

The structure factors $S(q)$ calculated from the X-ray and neutron diffraction data show very good coincidence within the whole $q$-range. Slight deviations for $0 \leqq q \leqq 1.7 \AA^{-1}$ are discussed but yield no hint at all on electron correlations independent of the nuclei.

\section{Introduction}

For the investigation of the atomic structure of molten metals and amorphous solids thermal neutron diffraction and X-ray diffraction methods are used. Both methods yield comparable results concerning the quality of the diffraction patterns. Thus during the investigation of binary systems results obtained with both methods are combined in order to determine partial structure factors. This combination only works on the assumption that both methods yield the same result if applied to a molten element.

Since X-rays are scattered by electrons, the determination of the atomic arrangement within a melt by an X-ray diffraction experiment necessitates the knowledge of the spatial distribution of the electrons around the nucleus ("form factor").

With nearly all atoms this distribution is so strongly determined by the inner electrons that the electron distribution within the melt can be described with sufficient accuracy by that of the free atom. With light elements such as lithium the outer electrons also play an important role for the spatial distribution of all the electrons. Thus, during the evaluation of an X-ray diffraction experiment per-

* Institut Max von Laue - Paul Langevin, Grenoble, France.

Reprint requests to Prof. Dr. S. Steeb, Max-Planck-Institut für Metallforschung, Institut für Werkstoffwissenschaften. Seestraße 92. D-7000 Stuttgart 1, West Germany. formed with molten lithium using the electron distribution of a free atom, certain differences in comparison with the results of a corresponding neutron diffraction experiment should be expected.

To verify this, a neutron and an X-ray diffraction experiment were performed with molten lithium. Since for light elements the intensity of the inelastically scattered X-rays (Compton scattering) is comparable to that of the elastically scattered radiation, the former had to be measured separately in order to correct properly the total measured X-ray intensity.

\section{Theoretical basis}

The atomic structure of a monoatomic melt is described by the pair correlation function $Q(r)$ which represents the mean number of atoms per unit volume at the distance $r$ from a reference atom. For large $r, \varrho(r)$ approaches $\varrho_{0}$, which is the mean number density of the melt. The corresponding variable in reciprocal space is the structure factor $S(q)$. The coherent scattering cross-section $\left.\frac{\mathrm{d} \sigma}{\mathrm{d} \Omega}\right|_{\text {coh }}$ for neutrons which are scattered coherently from a molten element is directly proportional to $S(q)$ :

$$
\left.\frac{\mathrm{d} \sigma}{\mathrm{d} \Omega}\right|_{\mathrm{coh}}=N \frac{\sigma_{\mathrm{coh}}}{4 \pi} S(q)
$$


with

$\sigma_{\text {coh }}=$ coherent scattering cross section per atom,

$N=$ number of atoms irradiated,

$q=\frac{4 \pi}{i} \sin \Theta$,

$i=$ wavelength,

$2 \Theta=$ scattering angle.

In contrast to the neutrons the X-rays are scattered from the electron core of the atoms. Therefore the scattering law also contains information concerning the electron distribution. The coherently scattered intensity of X-rays $I_{\text {coh }}^{\mathrm{X}}$ obtained with a system of identical atoms can be obtained in electron units according to

$$
I_{\mathrm{coh}}^{\mathrm{X}}=N f^{2}(q) S(q) \text {. }
$$

The form factor $f(q)$ means the Fourier transformation of the electron distribution around the atomic nucleus. To obtain (2) the so called rigid bond approach has to be used, i.e. one assumes that all electrons can be attributed to the nucleus in a definite way and are rigidly bonded. For practical use one proceeds one step further and applies for the evaluation of (2) the form factor of the free atom as calculated by the Hartree-Fock method [1].

Thus the assumption is made that the electron distribution around the nucleus within the melt is the same as that within the gas. For metallic melts these assumptions are only valid for the inner electrons. The conduction or valency electrons are delocalized and therefore must be treated separately. Egelstaff et al. [2] have derived for this case the equation

$$
\begin{gathered}
I_{\mathrm{coh}}^{\mathrm{X}}=N\left[f_{\mathrm{i}}^{2}(q) S_{\mathrm{ii}}(q)+2 n f_{\mathrm{i}}(q) S_{\mathrm{iv}}(q)\right. \\
\left.+n^{2} S_{\mathrm{vv}}(q)\right] .
\end{gathered}
$$

Equation (3) has the same structure as the scattering law of a binary alloy with the partial structure factors $S_{\mathrm{ii}}, S_{\mathrm{iv}}$, and $S_{\mathrm{vv}}$. The components in the present case are the ions $\mathrm{i}$ and the free or valency electrons $\mathrm{v}$ of the molten element. $f_{\mathrm{i}}(q)$ means the form factor of the inner electrons, $n$ the number of free electrons per atom. $S_{\mathrm{ii}}(q) \equiv S(q)$ describes the correlations between the ions, $S_{\mathrm{vv}}$ the correlations between the valency electrons, and $S_{\mathrm{iv}}$ the cross correlation between ions and free electrons.
Egelstaff et al. [2] propose to determine the three partial structure factors $S_{\mathrm{ii}}, S_{\mathrm{iv}}$, and $S_{\mathrm{vv}}$ by a combination of three diffraction experiments. A neutron diffraction experiment yields directly $S_{\mathrm{ii}}$. An X-ray diffraction experiment and an electron diffraction experiment for which a scattering law corresponding to (3) is valid should yield $S_{\mathrm{iv}}$ and $S_{\mathrm{vv}}$. As was shown recently [3] such a "three beam experiment" cannot be performed since electron diffraction experiments are inconvenient for combination with the two other radiations.

A further point is that with all metals except lithium and beryllium the number of valency electrons and therefore their contribution to the scattering is small compared to that of the inner electrons. Furthermore the valency electrons are not completely delocalized but more or less concentrated around the metal ions. For these reasons this delocalizing had never so far been observed in diffraction experiments. In the literature there are studies and speculations concerning the question about the shape of $S_{\mathrm{vv}}(q)$, for example in alkali metals $[4,5,6]$.

In the present work we have tried to find out whether there are any differences to be shown up experimentally between a neutron experiment and an X-ray experiment which latter should be evaluated using the form factor of a free atom according to (2). As a specimen the molten element lithium was chosen, since only for beryllium the ratio between the number of valency electrons and

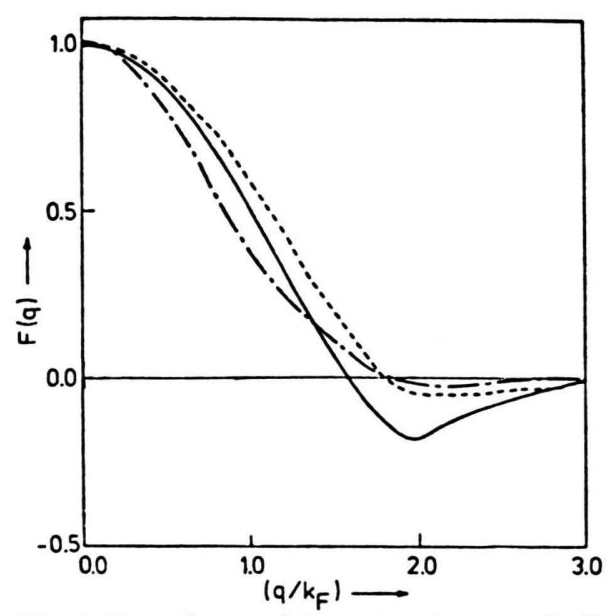

Fig. 1. Form factor of the outer electron of a lithium atom. a) Crystalline lithium; — according to [7]; -.- according to [8]. b) Free lithium atom; $-\cdot \cdot \cdot-$ according to [7]. 
the number of inner electrons is larger. Lithium, however, can be handled in molten state much better than beryllium.

Using Fig. 1 the effect to be expected can be estimated. It shows the form factor of the valency electrons of crystalline lithium as calculated according to the rigid bond approach by Visser et al. [7] and Perrin et al. [8] in comparison to the form factor of the $2 \mathrm{~s}$-wave function of the free lithium atom. To estimate the order of magnitude of the deviation in the scattering experiment it must be taken into consideration that the form factor of the inner electrons must be added to that of the outer electrons.

\section{Experiments}

\section{Neutron diffraction with molten lithium}

For the neutron diffraction experiment we used lithium containing $99.05 \%{ }^{7} \mathrm{Li}$ and $0.95 \%{ }^{6} \mathrm{Li}$. The metal was filled under high purity argon into a cylindrical container and sealed by electron beam welding. The can was made from $0.1 \mathrm{~mm}$ vanadium foil and had an external diameter of $11.4 \mathrm{~mm}$. The neutron diffraction experiments were carried out at 470,575 , and $725 \mathrm{~K}$ on the instrument D4 at the Institut Laue-Langevin, using the same counting strategy and almost the same experimental set-up as described by Ruppersberg and Reiter [9]. Only the vacuum furnace was replaced by a helium-filled oven which is more suitable at lower temperature.

Using a wavelength of $0.695 \AA$, the scattered intensity was measured in $2 \Theta$ steps of about $0.2^{\circ}$ for $2 \Theta=1.35^{\circ}$ to $90^{\circ}\left(q=0.21-12.8 \AA^{-1}\right) . I_{\mathrm{s}}(q)$ given in Table 1 is the number of neutrons collected per step which were scattered from the melt, including coherent, incoherent, and multiple diffraction. $I_{\mathrm{t}}(q)$ is the corresponding number for the experimental run of the sample in the furnace.
The data reduction was performed as explicitly described by Ruppersberg and Reiter [9]. The data were normalized so as to yield the correct $S(0)$ values (Table 1) and to make $q(S(q)-1)$ modulate at large $q$ uniformly about zero. The incoherent cross section $\sigma_{i}$ was assumed to be an adjustable parameter. The observed values are given together with values from the literature in Table 1 . The observed differences are small. Table 2 shows the values of $\pm \Delta q(S(q)-1)$ corresponding to the possible deviations of $q(S(q)-1)$ due to the normalization procedure. At the first peak of $S(q)$ the uncertainty related to the normalization procedure is much larger than the effect of counting statistics.

The Fourier transforms $r\left(\varrho(r)-\varrho_{0}\right)$ of the $q(S(q)-1)$ curves are shown in Figure 2. At small $r, \varrho(r)=0$ and the curves should be straight lines. To detect the origin in $q$ space of the corresponding deviations, the latter were backtransformed. The resulting $\Delta^{\prime} q(S-1)$ values are given

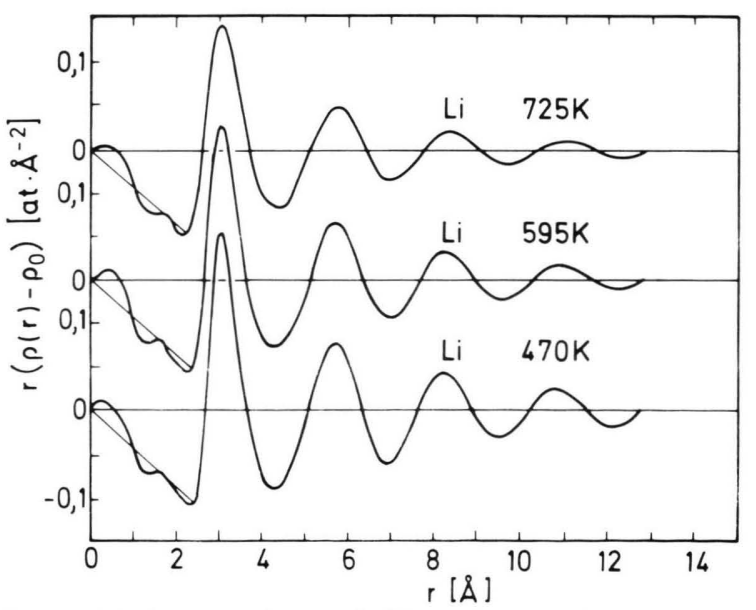

Fig. 2. Lithium, molten: $r\left(\varrho(r)-\varrho_{0}\right)$ according to neutron diffraction experiments for three different temperatures.

Table 1. Temperature $T$, number-density $\varrho_{0}, S(0)$ and scattering cross-sections of the investigated melts. $I_{\mathrm{t}}(q)$ is the total number of neutrons collected at this $q$-value. $I_{\mathrm{s}}(q)$ is the number of neutrons coming from the melt.

\begin{tabular}{|c|c|c|c|c|c|c|c|c|c|c|c|}
\hline \multirow[t]{2}{*}{ Substance } & \multirow{2}{*}{$\begin{array}{l}T \\
{[\mathrm{~K}]}\end{array}$} & \multirow{2}{*}{$\begin{array}{l}10^{3} \cdot Q_{0} \\
{\left[\text { at } \cdot \AA^{-3}\right]}\end{array}$} & \multirow[t]{2}{*}{$10^{4} \cdot S(0)$} & $\sigma_{\mathrm{c}}$ & $\sigma_{\mathrm{m}}$ & $\sigma_{\mathrm{i}}$ & \multirow[t]{2}{*}{$\sigma_{i}$ exp. } & $I_{\mathrm{t}}(0.8)$ & $I_{\mathrm{S}}(0.8)$ & $I_{\mathrm{t}}(7)$ & $I_{\mathrm{S}}(7)$ \\
\hline & & & & \multicolumn{3}{|l|}{ [barns] } & & \multicolumn{4}{|c|}{ [ $10^{3}$ neutrons] } \\
\hline $\begin{array}{l}\mathrm{Li} \\
\mathrm{Li} \\
\mathrm{Li}\end{array}$ & $\begin{array}{l}470 \\
595 \\
725\end{array}$ & $\begin{array}{l}44.5 \\
43 \\
42\end{array}$ & $\begin{array}{l}306 \\
408 \\
525\end{array}$ & $\begin{array}{l}0.61 \\
0.61 \\
0.61\end{array}$ & $\begin{array}{l}0.07 \\
0.07 \\
0.07\end{array}$ & $\begin{array}{l}0.83 \\
0.83 \\
0.83\end{array}$ & $\begin{array}{l}0.90 \\
0.92 \\
0.87\end{array}$ & 46 & 20 & 43 & 30 \\
\hline
\end{tabular}


in Table 2. These values which correspond in all three cases to a long wavelength error were added at $q>1.7 \AA^{-1}$ to the original $q(S(q)-1)$ to give the corrected curves, for which the position $q$ and the amplitude of the maxima and minima are also collected in Table 2. The spurious oscillations remaining in the recalculated $r\left(\varrho(r)-\varrho_{0}\right)$ curves are almost invisible and also the termination errors are very small. Short wavelength errors in $q(S(q)-1)$ cannot be detected by this method because the deviations in $r$-space are only visible for small $r$-values. However, we see no experimental reasons for strongly localized errors. The maximum values of $\Delta^{\prime} q(S(q)-1)$ in Table 2 correspond to a 1 to $2 \%$ deviation of the intensity $I_{\mathrm{t}}(q)$ which might, for example, be due to some misalignment of the sample with respect to the goniometer axis. Tables of $S(q), q(S(q)-1)$ and of its Fourier transform may be obtained from one of the authors (H.R.).

$S(q)$ calculated from the individually measured intensities is given for $q<2 \AA^{-1}$ in Figure 3. The full curves in this figure indicate the $95 \%$ confidence limits. They were calculated assuming known $S(0)$ values and the following scattering law: $S(q)=S(0)+a \cdot q^{2}$ which seems to be a useful approximation for $q<1.6 \AA^{-1}$. The circles in the $595 \mathrm{~K}$ graph are values obtained previously by Ruppersberg and Egger [10] with the same D4 diffractometer before the single counter was replaced by the multicounter. The new $q(S(q)-1)$ curve has a somewhat higher first peak and lower second minimum. The deviations are +0.05 and -0.15 , respectively.

$\varrho(r)$ and $S(q)$ of liquid lithium have been calculated by Jacucci et al. [11] using molecular dynamics and using a pair potential elaborated by Rasolt and Taylor [12] and Dagens et al. [13]. In Table 3 calculated $\left(\varrho(r) / \varrho_{0}-1\right)$ and $(S(q)-1)$ values are compared with our experimental data and the agreement is seen to be excellent. Hafner [14] performed an ab initio calculation of $S(q)$ and of various thermodynamic properties for several liquid metals including Li starting from a hard sphere system with hard sphere diameters obtained from optimized pseudo potentials and using a variational thermodynamic perturbation scheme. The agreement with experiment (Table 3 ) is remarkably good but less good than in the case of computer simulation.

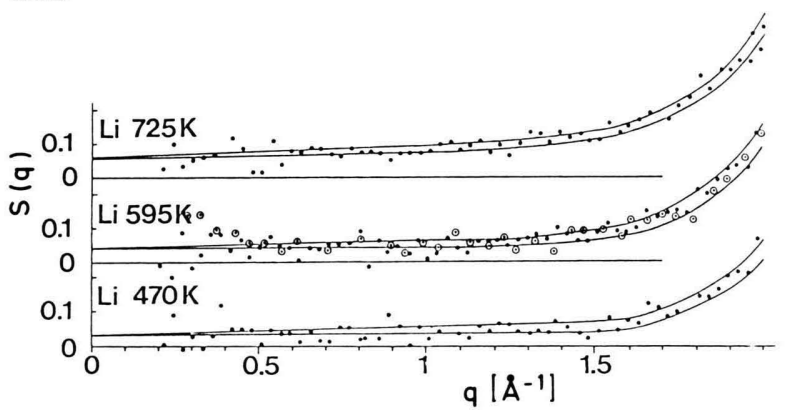

Fig. 3. Lithium, molten: $S(q)$. Points: Calculated from individually measured intensities. Circles: from Ruppersberg and Egger [10]. Continuous lines: $95 \%$ confidence limits assuming given $S(0)$.

Table 2. Position and amplitudes of the maxima and minima of $q(S(q)-1)$. Error due to normalization: $\Delta q(S-1)$. Correction from backtransformation of oscillations at small $r$ in the $r\left(\varrho(r)-\varrho_{0}\right)$-curves of Fig. $2: \Delta^{\prime} q(S-1)$.

\begin{tabular}{|c|c|c|c|c|c|c|c|c|}
\hline & $1^{\mathrm{st}} \min$ & $1^{\mathrm{st}} \max$ & $2^{\text {nd }} \min$ & $2^{\text {nd }} \max$ & $3^{\text {rd }} \min$ & $3^{\text {rd }} \max$ & $4^{\text {th }} \min$ & $4^{\text {th }} \max$ \\
\hline $\begin{array}{l}\mathrm{Li}, 470 \mathrm{~K} \\
q\left(\AA^{-1}\right) \\
q(S-1) \\
\pm \Delta q(S-1) 10^{2} \\
\Delta^{\prime} q(S-1) \cdot 10^{2}\end{array}$ & $\begin{array}{rl} & 1.85 \\
-1.59 & 2 \\
-8\end{array}$ & $\begin{array}{l}2.47 \\
5.06 \\
10 \\
-20\end{array}$ & $\begin{aligned} & 3.45 \\
&- 1.34 \\
& 3 \\
&-16\end{aligned}$ & $\begin{array}{r}4.60 \\
1.07 \\
5 \\
-13\end{array}$ & 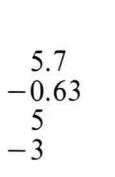 & $\begin{array}{l}6.8 \\
0.40 \\
5 \\
+6\end{array}$ & $\begin{array}{l}8.0 \\
-0.19 \\
6 \\
-1\end{array}$ & $\begin{array}{l}9.0 \\
0.14 \\
7 \\
-4\end{array}$ \\
\hline $\begin{array}{l}\mathrm{Li}, 595 \mathrm{~K} \\
q\left(\AA^{-1}\right) \\
q(S-1) \\
\pm \Delta q(S-1) 10^{2} \\
\Delta^{\prime} q(S-1) \cdot 10^{2}\end{array}$ & $\begin{array}{c}1.80 \\
-1.53 \\
3 \\
-8\end{array}$ & $\begin{array}{c}2.46 \\
4.11 \\
10 \\
-24\end{array}$ & $\begin{aligned} & 3.50 \\
- & 1.22 \\
& 4 \\
- & 21\end{aligned}$ & $\begin{array}{r}4.65 \\
0.94 \\
5 \\
-12\end{array}$ & $\begin{array}{l}5.65 \\
-0.48 \\
6 \\
0\end{array}$ & $\begin{aligned} & 6.8 \\
& 0.26 \\
& 6 \\
+ & 10\end{aligned}$ & $\begin{array}{l}8.1 \\
-0.14 \\
7 \\
0\end{array}$ & $\begin{array}{l}9.3 \\
0.08 \\
8 \\
-2\end{array}$ \\
\hline $\begin{array}{l}\mathrm{Li}, 725 \mathrm{~K} \\
q\left(\AA^{-1}\right) \\
q(S-1) \\
\pm \Delta q(S-1) 10^{2} \\
\Delta^{\prime} q(S-1) \cdot 10^{2}\end{array}$ & $\begin{aligned} & 1.78 \\
&-1.40 \\
& 3 \\
&-8\end{aligned}$ & $\begin{aligned} & 2.45 \\
& 3.23 \\
& 10 \\
&-19\end{aligned}$ & $\begin{array}{r}3.45 \\
-0.96 \\
4 \\
-17\end{array}$ & $\begin{array}{l}4.55 \\
0.68 \\
5 \\
-8\end{array}$ & $\begin{array}{l}5.7 \\
-0.37 \\
6 \\
2\end{array}$ & $\begin{array}{l}6.9 \\
0.14 \\
6 \\
5\end{array}$ & $\begin{array}{l}8.1 \\
-0.07 \\
6 \\
-1\end{array}$ & $\begin{array}{l}9.1 \\
0.03 \\
5 \\
-1\end{array}$ \\
\hline
\end{tabular}


Table 3. Position and amplitude of the maxima, minima, and zero points of $S(q)-1$ and $\varrho(r) / \underline{Q}_{0}-1$ obtained experimentally for $\mathrm{Li}$ at $595 \mathrm{~K}$ and calculated by Jaccuci et al. [11]: $J$ as well as Hafner [14]: $H$.

\begin{tabular}{|c|c|c|c|c|c|c|c|c|c|c|}
\hline & \multicolumn{3}{|c|}{$q\left[\AA^{-1}\right]$} & \multicolumn{3}{|c|}{$S(q)-1$} & \multicolumn{2}{|l|}{$r[\AA]$} & \multicolumn{2}{|c|}{$\left(\underline{Q}(r) / \varrho_{0}\right)$} \\
\hline & \multirow[t]{2}{*}{$\exp$} & \multicolumn{2}{|c|}{ calculated } & \multirow[t]{2}{*}{$\exp$} & \multicolumn{2}{|c|}{ calculated } & \multirow[t]{2}{*}{$\exp$} & \multirow{2}{*}{$\begin{array}{l}\text { calc } \\
J\end{array}$} & \multirow[t]{2}{*}{$\exp$} & \multirow{2}{*}{$\begin{array}{l}\text { calc } \\
J\end{array}$} \\
\hline & & $J$ & $H$ & & $J$ & $H$ & & & & \\
\hline zero & 2.17 & 2.15 & 2.15 & 0 & 0 & 0 & 2.63 & 2.62 & 0 & 0 \\
\hline $1^{\mathrm{st}} \max$ & 2.45 & 2.47 & 2.50 & 1.68 & 1.67 & 1.25 & 3.03 & 3.05 & 1.33 & 1.30 \\
\hline zero & 2.90 & 2.90 & 3.00 & 0 & 0 & 0 & 3.63 & 3.60 & 0 & 0 \\
\hline $1^{\mathrm{st}} \min$ & 3.40 & 3.43 & 3.45 & -0.36 & -0.35 & -0.30 & 4.20 & 4.25 & -0.40 & -0.40 \\
\hline zero & 4.12 & 4.07 & 4.15 & 0 & 0 & 0 & 5.05 & 5.00 & 0 & 0 \\
\hline $2^{\text {nd }} \max$ & 4.60 & 4.57 & 4.70 & 0.20 & 0.18 & 0.25 & 5.65 & 5.64 & 0.26 & 0.27 \\
\hline zero & 5.17 & 5.15 & & 0 & 0 & 0 & 6.32 & 6.36 & 0 & 0 \\
\hline $2^{\text {nd }} \min$ & 5.60 & 5.65 & & -0.08 & -0.1 & & 6.9 & 6.9 & -0.14 & -0.17 \\
\hline zero & 6.35 & 6.35 & & 0 & 0 & & 7.65 & 7.65 & 0 & 0 \\
\hline $3^{\text {rd }} \max$ & 6.85 & 6.8 & & 0.04 & 0.04 & & 8.2 & 8.2 & 0.09 & 0.1 \\
\hline zero & 7.55 & & & 0 & 0 & & 8.95 & 9.9 & 0 & 0 \\
\hline
\end{tabular}

\section{$X$-ray diffiaction with molten lithium}

The X-ray diffraction experiment was performed using a $12 \mathrm{~kW}$ rotating anode generator using molybdenum radiation. The characteristic Mo-K $\alpha$ line was selected by a graphite-monochromator and focussed onto the detector slit. According to Fig. 4 the experiment was done using focussing transmission geometry. The specimen consisted of a lithium layer $1 \mathrm{~mm}$ thick between two Be-single crystal windows, each of them $0.3 \mathrm{~mm}$ thick. The container was built up using a sandwich construction whereby the Be-single crystals were adjusted in such a way that no Be-reflexes could reach the counter. The furnace allowed scattering angles $2 \Theta$ up to $70^{\circ}$ using the $\Theta-2 \Theta$ mode. Two measurements were performed, one with the specimen inside the container $\left(5 \cdot 10^{4}\right.$ pulses per point $)$ and one with the empty container $\left(2 \cdot 10^{4}\right.$ pulses per point).

The temperature during the measurement was $12 \pm 2^{\circ}$ above the melting point of lithium. In spite of very careful adjustment and mounting of the beryllium single crystals during heating small Bragg reflexions were observed which were eliminated graphically. Figure 5 shows the rough data obtained from the X-ray diffraction experiment.

The corrections for container scattering, absorption within the specimen and the Be-windows, and polarization were performed according to the usual methods. Normalizing was done according to Krogh-Moe [15, 16]. Considerable difficulties arose during the correction for inelastic scattering. Since there is no possibility of experimentally eliminating this radiation within the total $q$-region (see [17]) the correction normally must be done according to data from literature. During this procedure one uses data which are calculated from Hartree-Fock wave functions for the free atom similarly to the form factor. This method works without problems for most elements since the inelastic scattering only forms a small contribution to the total scattering. With light elements however, there is a totally different situation since the ratio between elastic and inelastic scattering is porportional to the atomic number.

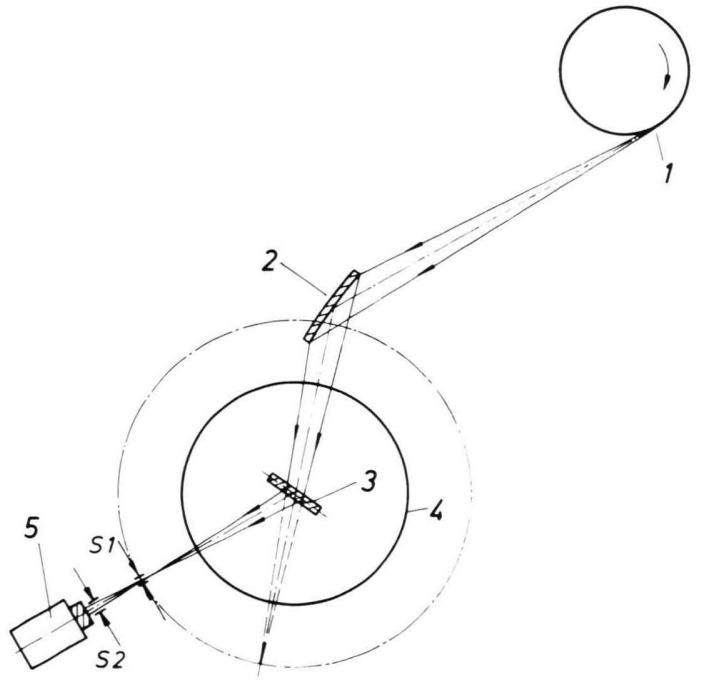

Fig. 4. Diffractometer for the X-ray diffraction experiment.

1 - Rotating anode generator

2 - Monochromator

3- Specimen

4 - Vacuum chamber

5 - Detector

S1, S2 - Slits 


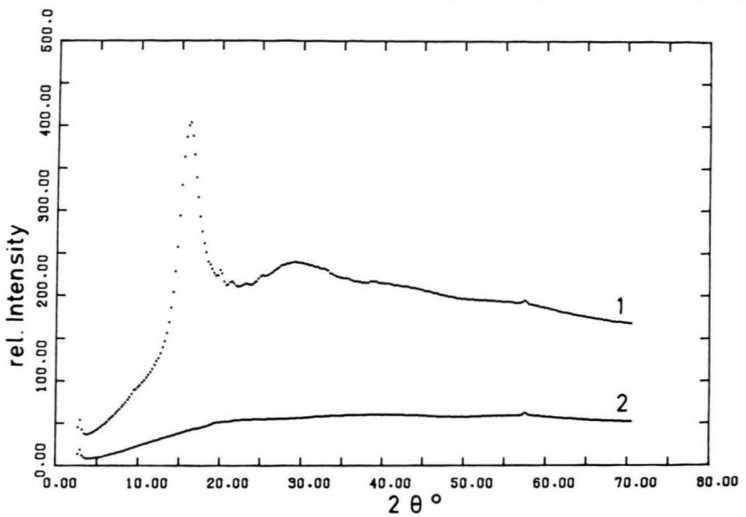

Fig. 5. X-ray diffraction experiment. Rough data. 1 - Molten lithium within the container, 2 - Empty container.

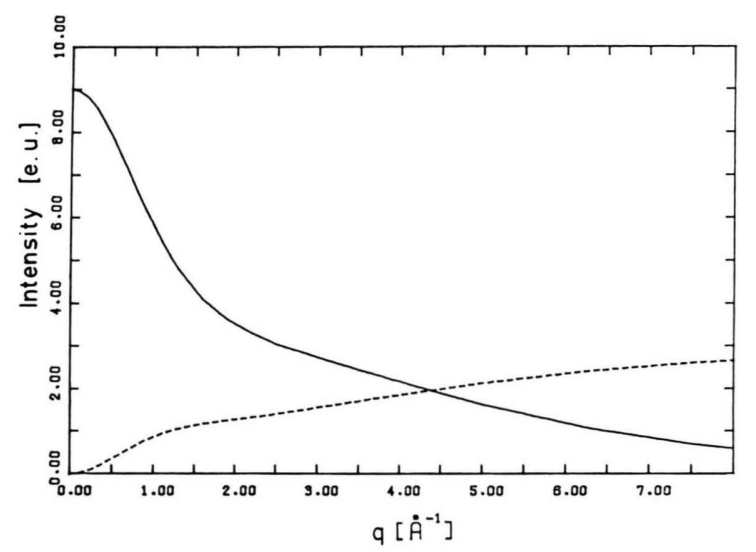

Fig. 6. Free lithium atom. - Elastic scattered X-ray intensity according to Ref. [1]; -..- Inelastic scattered $\mathrm{X}$-ray intensity according to Ref. [1].

Figure 6 shows for the free Li-atom the squared form factor compared to the inelastic scattering. As is known from experiments performed with crystalline beryllium [18] especially in the region $q<2 k_{\mathrm{f}}$ there exist pronounced deviations of the inelastic intensity between that of the free atom and that of the condensed phase. Since for Li there exist no measured data the inelastic scattering had to be measured separately.

\section{Experimental determination of the inelastically scattered $X$-ray intensity}

The simplest case of an inelastic X-ray scattering process is the elastic impact of an X-ray quantum to a free electron at rest. The X-ray quantum thereby loses energy and its wavelength is varied. The variation $\Delta i_{0}$ of the wavelength amounts to

$$
\Delta i_{0}=2 i_{c} \sin ^{2} \Theta=\left(i_{c} / 8 \pi^{2}\right) i^{2} q^{2}
$$

with

$$
i_{\mathrm{c}}=h / m c=\text { Compton wavelength . }
$$

Since the electrons in reality are not at rest but have a certain velocity distribution, according to this simple concept a broad line around the mean wavelength shift $\Delta i_{0}$ can be expected in the inelastic $\mathrm{X}$-ray spectrum. According to (4) at small scattering angles $\Delta \lambda_{0}$ varies proportional to $\theta$. With finite resolution of the spectrometer the inelastic line can therefore not be separated from the elastic line at small scattering angles.

The main problem with the measurement of the inelastic scattering of X-rays is due to the very small intensity. In the past, nearly all of the measurements were therefore performed without a primary beam monochromator. During these experiments only the scattered radiation was analyzed within a crystal spectrometer working in step scan mode. Scattered radiation arising from the Bremsstrahlung thereby forms a background which disturbs the measurements (compare [8]).

Using a position sensitive detector during the present work a novel spectrometer was constructed and installed. This instrument makes it possible to record the total energy spectrum at once. Thus the measuring time can be reduced drastically and a primary beam monochromator can be applied.

Figure 7 shows the principle of the spectrometer. The beam produced within the rotating anode $\mathrm{X}$-ray generator (1) is monochromatized by the monochromator (2) and is analyzed according to the energy by a curved analyzing crystal (4) after being scattered at the specimen (3). Using this method, radiation of different energy enters the position sensitive detector (5) at different positions. To avoid air-absorption and air-scattering the total beam geometry is kept in vacuum or in helium.

The spectrometer is mounted on a turnable plate with the specimen mounted in the centre. The scattering angle can be varied in the region $0^{\circ} \leqq 2 \Theta \leqq 150^{\circ}$. Table 4 contains the characteristic data of the spectrometer. A similar instrument, however using photographic film instead of a position sensitive detector (PSD) was used by du Mond [19]. A spectrometer using a PSD as de- 


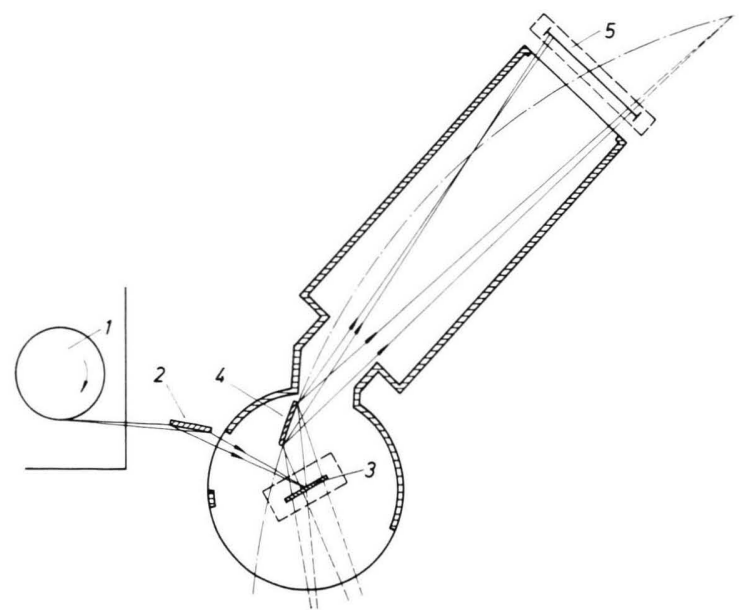

Fig. 7. Compton spectrometer. Schematic diagram.

1 - Rotating anode X-ray generator,

2 - Monochromator,

3-Specimen,

4- Analyzer,

5 - Position sensitive detector.

scribed above was used by Loupias et al. [20] and by Pattison et al. [21].

In contrast to the X-ray elastic diffraction experiment the determination of the inelastic X-ray scattering was performed using $\mathrm{Cu}-\mathrm{K} \alpha$ radiation, since the energy resolution of a crystal with a given mosaic spread increases with increasing wavelength. The presumption for this procedure is the fact that the scattering intensity only depends on the momentum transfer $q=(4 \pi / \lambda) \sin \Theta$ and not on the quantum energy of the primary beam. This presumption is fulfilled for lithium caused by the small binding energy of the electrons. The specimen for the inelasic experiment consisted of a lithium platelet $1 \mathrm{~mm}$ thick with a welded container. The windows were Ni-foil $6 \mu \mathrm{m}$ thick. Nickel was used since it shows a minimum absorption for $\mathrm{Cu}-\mathrm{K} \alpha$ radiation and furthermore can be obtained as thin

Table 4. Data of the spectrometer.

\begin{tabular}{ll}
\hline Monochromator & Quartz $110 \overline{1}$ 1st order \\
Primary wavelength & Cu-K $\alpha_{1}, i=1.54 \AA$ \\
Suppression of $\alpha_{2}$ & $I_{\alpha_{2}} / I_{\alpha_{1}}<0.1$ \\
Analyzing crystal & Quartz $110 \overline{1}$ 2nd order \\
Simultaneously detectable & \\
wavelength region & $1.5<i<1.7 \AA$ \\
Energy resolution & $E / \Delta E=10^{3}$ \\
Scattering angle & $0^{\circ}<2 \Theta<150^{\circ}$
\end{tabular}

foil and yields no inelastic scattering so that the investigation of the empty container was not necessary. The measurement was performed using solid lithium since the nickel foil and molten lithium showed chemical reactions and since no difference in the inelastic scattering between the solid and the liquid phase was to be expected. The corrections for absorption and polarization were performed by analogy with the procedure used for the X-ray diffraction experiment. A difficulty arose from the high wavelength dependence of the transmission of the nickel foil. Using an EXAFS apparatus (see Ref. [22]) the transmission was determined and used during the correction procedure.

Figure 8 shows three typical results from three different regions of the scattering angle. The curves are corrected for background, absorption, polarization, and relativistic effects (Breit-Dirac-factor). The upper figure for $2 \Theta=70^{\circ}$ shows besides the elastic line $(i=1.54 \AA)$ and the broadened inelastic
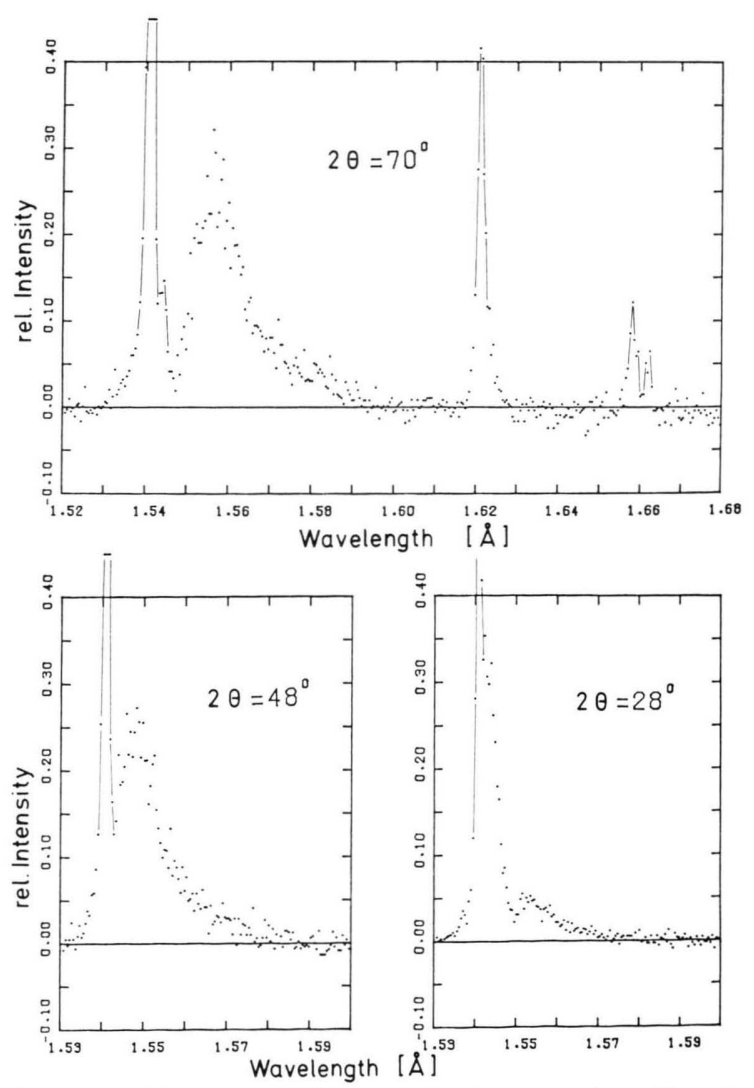

Fig. 8. Lithium, crystalline: Inelastic scattering (Results typical for the corresponding region of scattering angle). 


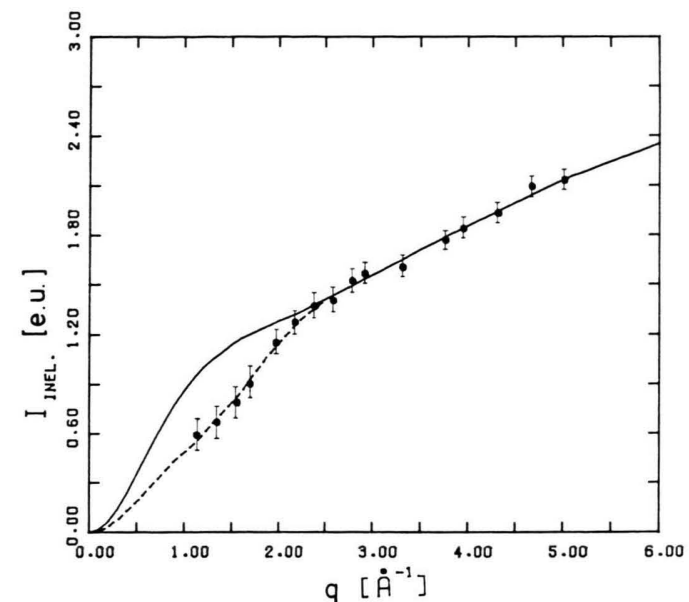

Fig. 9. Lithium, crystalline: Integral inelastic scattering: --- or - Experimental result; — Intensity calculated for the free atom.

spectrum a $\mathrm{Co}-\mathrm{K} \beta$ line $(\lambda=1.62 \AA)$ and a $\mathrm{Ni}-\mathrm{K} \alpha$ line $(\lambda=1.66 \AA)$. These fluorescence lines arise from the nickel foil which contains about $8000 \mathrm{ppm}$ Co. For $2 \Theta>60^{\circ}$ the elastic and the inelastic line are separated. To obtain the integral inelastic scattered intensity the integration must be performed via the corresponding wavelength region. Already in the region $35^{\circ}<2 \Theta<60^{\circ}$ an overlap of the residuals of the $\alpha_{2}$-line and the inelastic intensity is observed. In this region the $\alpha_{1}$-intensities measured at large angles are fitted to the corresponding line. Hereafter the experimental curve is integrated and the integral intensity of the $\alpha_{1}$ and $\alpha_{2}$ lines of the fitted reference curve is subtracted. In the region $2 \Theta<35^{\circ}$ the procedure is in principle the same. However the fitting process is not so precise since only the left flange of the $\alpha_{1}$ line can be used which causes the main error.

For the normalization of the experimental values a curve calculated for the free atom was used, since for $q>3 \AA^{-1}$ no deviation from this curve was to be expected. Figure 9 shows the result of the measurement of X-rays inelastically scattered with solid lithium using a measuring time of $4 \mathrm{~h}$ per point. The full line shows the values calculated for the lithium atoms according to [1]. Distinct deviations are observed for $q<2 k_{\mathrm{f}}=2.3 \AA^{-1}$. This result is consistent with the measurements performed with beryllium in [18]. The dashed line in Fig. 9 shows the curve which was finally used for the correction of the X-ray diffraction experiment.

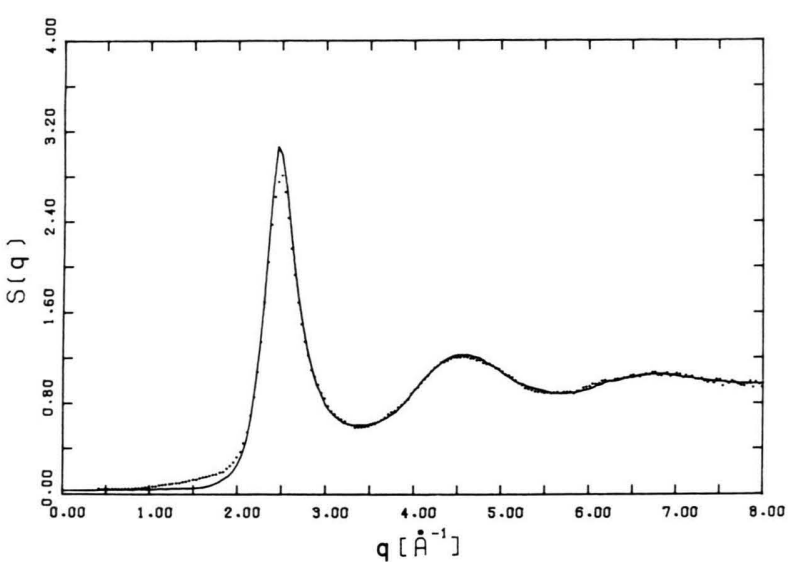

Fig. 10. Lithium, molten: Structure factors: $\cdots$ X-ray diffraction; - Neutron diffraction.

\section{Results and Discussion}

The dotted line in Fig. 10 shows the structure factor of molten lithium calculated from the X-ray diffraction data using the form factor of the free atom. The correction of the experimental values for inelastic scattering was done using the data from Figure 9. For comparison the structure factor from the neutron diffraction experiment is also plotted. At $q>2 \AA^{-1}$ the deviations between the two curves are smaller than $5 \%$. For $q<1 \AA^{-1}$, where the height of the structure factor is very small and where the inelastic part only could be assumed, the differences are smaller than $10 \%$. Within these two regions the differences are not larger than the experimental error.

Attention should be drawn, however, to the region $1<q<2 \AA^{-1}$ where a rather large difference between the two curves is observed, which may be caused by the following factors:

i) A hidden systematic error, especially during the measurement of the inelastic intensity, which only could be recognized by an independent repetition of the measurement.

ii) The difference which was determined for solid lithium arises from a variation of the inelastic scattering on melting. However, a control measurement performed with molten lithium for $q<2 \AA^{-1}$ excludes this possibility.

iii) The neutron curve is too low. In fact, according to Table 2 the correction for the spurious 
oscillations in $\left(\varrho(r)-\varrho_{0}\right)$ decreased the original $S$ (1.85) by 0.05 which is about the distance between the X-ray and the neutron curve in Figure 10. There might in principle be some higher frequency terms reducing this correction in the $q$-range between 1.7 and $2 \AA^{-1}$. However, this leaves unexplained the deviation at $q<1.7 \AA^{-1}$ where the original neutron data $I_{\mathrm{s}}(q)$ vary a little with $q$ as shown in Figure 10 .

iv) The difference between the neutron and the $\mathrm{X}$-ray curve corresponds to a form factor for molten lithium showing a pronounced maximum at $q=1.5 \AA^{-1}$. Such a maximum seems very improbable since it would correspond to an electron density distribution which would not decrease monotonically from the centre.

[1] J. H. Hubbel, Wm. J. Veigele, E. A. Briggs, R. T. Brown, J. T. Cromer, and R. J. Howerton, J. Phys. Chem. Ref. Data 4, 471 (1975).

[2] P. A. Egelstaff, N. H. March, and N. C. McGill, Can. J. Phys. 52, 1651 (1974).

[3] F. Paasche, H. Olbrich, U. Schestag, and S. Steeb, Z. Naturforsch. 37 a, 1215 (1982).

[4] S. Cusack, N. H. March, M. Parrinello, and M. P. Tosi, J. Phys. F6, 749 (1976).

[5] N. H. March and M. P. Tosi, Phys. Chem. Liq. 10, 113 (1980).

[6] P. J. Dobson, J. Phys. C11, L 295 (1978).

[7] E. G. Visser, W. Geertsma, W. van der Lugt, and J. Th. M. de Hosson, Z. Naturforsch. 35 a, 373 (1980).

[8] R. C. Perrin, R. Taylor, and N. H. March, J. Phys. F5, 1490 (1975).

[9] H. Ruppersberg and H. Reiter, J. Phys. F. 12, 1311 (1982).

[10] H. Ruppersberg and H. Egger, J. Chem. Phys. 63, 4095 (1973).
Thus, within the region $1<q<2 \AA^{-1}$ discrepancies exist which are not fully understood. However, within the main part of the $q$-region no discrepancy between the X-ray and the neutron-diffraction experiment is observed. In particular there are no experimental hints on electron correlations independent of the nuclei as was assumed, for example, by Dobson [6].

\section{Acknowledgements}

Thanks are due to the Deutsche Forschungsgemeinschaft for financial support.

[11] G. Jacucci, M. L. Klein, and R. Taylor, Solid State Comm. 19,657 (1976).

[12] M. Rasolt and R. Taylor, Phys. Rev. B11, 2717 (1975).

[13] L. Dagens, M. Rasolt, and R. Taylor, Phys. Rev. B11, 2726 (1975).

[14] J. Hafner, Phys. Rev. A 16, 351 (1977).

[15] W. Sperl, Diplom thesis, Universität Stuttgart 1980.

[16] M. J. Huijben, Thesis, Riksuniversiteit, Groningen 1978.

[17] W. Ruland, Brit. J. Appl. Phys. 15, 1301 (1964).

[18] W. Schülke, U. Berg, and O. Brümmer, Phys. Stat. Sol. 35, 227 (1969).

[19] B. Williams, Compton Scattering, McGraw Hill, London 1977.

[20] G. Loupias and J. Petiau, J. Physique 41, 265 (1980).

[21] P. Pattison, H.-J. Bleif, and J. R. Schneider, J. Phys. I 14, 95 (1981)

[22] F. Schmückle, Thesis, Universität Stuttgart 1981. 